\title{
Erratum to: Bland and tumor thrombi in abdominal malignancies: magnetic resonance imaging assessment in a large oncologic patient population
}

M.R. Engelbrecht, Oguz Akin, Devesh Dixit, Lawrence Schwartz

Department of Radiology, Memorial Sloan-Kettering Cancer Center, New York, NY, USA

Erratum to: Abdom Imaging (2010) DOI 10.1007/s00261-010-9608-6

In the author group the first author name was stated incorrectly as Marc Engelbrecht. The correct name is M.R. Engelbrecht. 